\title{
Aqueous Humor Mediator and Cytokine Aberrations in Diabetic Retinopathy and Diabetic Macular Edema: A Systematic Review and Meta-Analysis
}

\author{
Jingyang Wu, ${ }^{1}$ Yifan Zhong, ${ }^{1}$ Song Yue, ${ }^{1}$ Kaibo Yang, ${ }^{1}$ Guisen Zhang $\mathbb{D},{ }^{2}$ Lei Chen, \\ and Lei Liu $(\mathbb{D})^{1,3}$ \\ ${ }^{1}$ Department of Ophthalmology, The First Hospital of China Medical University, Shenyang 110001, China \\ ${ }^{2}$ Hohhot Chao Ju Eye Hospital, Hohhot 010000, China \\ ${ }^{3}$ Department of Public Service, The First Hospital of China Medical University, Shenyang 110001, China \\ Correspondence should be addressed to Guisen Zhang; zhangguisen76@sohu.com and Lei Liu; liuleijiao@163.com
}

Received 7 May 2019; Revised 20 October 2019; Accepted 30 October 2019; Published 23 November 2019

Academic Editor: Jacopo Gervasoni

Copyright ( 2019 Jingyang Wu et al. This is an open access article distributed under the Creative Commons Attribution License, which permits unrestricted use, distribution, and reproduction in any medium, provided the original work is properly cited.

\begin{abstract}
Purpose. To evaluate the relationship between the aqueous humor levels of VEGF, TNF- $\alpha$, IL-10, IL-6, IL-12, MCP-1, and IP-10 with DR/DME. Methods. PubMed, Web of Science, Embase, China National Knowledge Infrastructure (CNKI), and Wanfang databases were searched up to October 2018. Systematic review and meta-analysis were conducted. Results. 18 studies comprising 362 cases with DR (100 with DME) and 620 controls without DR were included in this meta-analysis. There was a significant association between VEGF levels in the aqueous humor and DR (standardized mean difference (SMD) 1.94 (95\% CI 1.05-2.83)) and DME (1.07 (0.71, 1.42)). Furthermore, a significant correlation was observed between levels of IL-6 and DR (3.53 $(0.37,6.69))$, and similarly correlation with DME $(1.26(0.30,2.21))$. The relationship between MCP-1 and DR and DME was significant, in which the SMD was $(0.49(0.09,0.89))$ and $(1.49(0.78,2.20))$, respectively. However, IL-12, IP- 10 , and TNF- $\alpha$ had no correlation with DR and DME, whereas there was a significant relationship between IL-8 and DME (1.68 (0.97, 2.40)). Conclusion. Elevated levels of VEGF, IL-6, and MCP-1 in the aqueous humor were associated with the risk for the presence of DR, and levels of VEGF, IL-6, IL-8, and MCP-1 were associated with the risk of DME. Furthermore, these biomarkers may be used as potential predictors or therapeutic targets for DR/DME.
\end{abstract}

\section{Introduction}

DR is one of the most common microcomplications of diabetes and has become the major cause of decreased vision and blindness in adults aged 20-74 years [1]. Recently, the disturbance of inflammatory reaction may play an important role in the numerous researches of the complex pathogenesis of DR [2]. Previous studies have shown that deregulation of immune responses associated with diabetes can induce high expression of various mediators resulting in the development of DR [3]. Furthermore, analysis of intraocular humors obtained from DR patients has indicated that some of the mediators (cytokines or chemokines) may be responsible for the pathogenesis of DR. The immune and inflammatory factors, e.g., VEGF, TNF- $\alpha$, IL-6, IL-8, and MCP-1, have been observed elevated in both aqueous humor and vitreous fluids in patients with DR $[4,5]$. Hence, the altered concentrations of various cytokines regarding these mechanisms may serve as important biomarkers to assess early detection or treatment of DR.

Diabetic macular edema (DME) is also one of the most serious causes of visual disability and blindness and affects approximately $14 \%$ of patients with diabetes [6]. The pathophysiology of DME is multifactorial, complicated, and partly still unknown. Several mediators, including VEGF, as well as other inflammatory cytokines, such as IL-6, IL-8, TNF- $\alpha$, IP-10, and MCP-1, are among the possible responsible mechanisms or are involved in the development of DME [7-9]. However, the exact cytokines in the aqueous humor regarding the pathogenesis of DME remain unknown. 


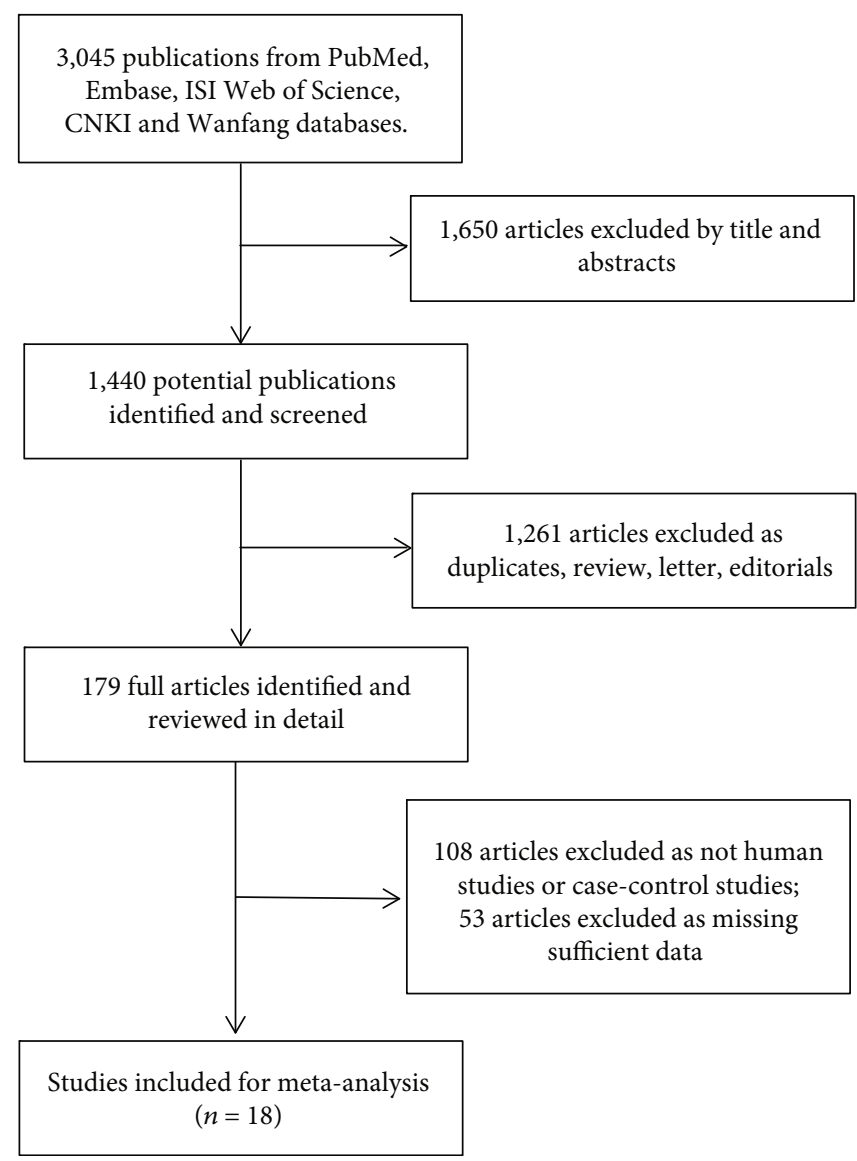

Figure 1: Flow diagram of study selection for the meta-analysis.

Taken together, the association between levels of inflammatory cytokines in the aqueous humor and DR is still controversial $[10,11]$. Moreover, there have been no systematic review and meta-analysis to evaluate the available evidence on the association of the aqueous humor levels of inflammatory cytokines with the risk of DR or DME. Herein, we perform this systematic review and meta-analysis to investigate the relationships between these biomolecule levels in the aqueous humor and the risk of DR and DME.

\section{Materials and Methods}

2.1. The Study Identification and Search Strategy. We identified the relevant evidences of mediators or cytokines in the aqueous humor among patients with type 2 diabetes mellitus (T2DM) by systematically searching PubMed, Embase, Web of Science, China National Knowledge Infrastructure (CNKI), and Wanfang databases, from inception to October 2018. The following key words were used to perform searches in the databases mentioned above: (inflammation, inflammatory markers, inflammatory biomarkers, inflammatory mediators, inflammatory cytokines) or (vascular endothelial growth factor, VEGF, tumor necrosis factor, TNF- $\alpha$, interleukin 6, IL-6, interleukin 8, IL-8, interleukin 10, IL-10, monocyte chemoattractant protein-1, MCP-1), and (diabetic retinopathy, retinopathy, DR) or (diabetic macular oedema, diabetic macular edema), and (aqueous humor, aqueous humour), without year restriction. Moreover, an extensive hand search was performed and the additional relevant studies were further reviewed in reference lists. The selection process was according to the Preferred Reporting Items for Systematic Reviews and Meta-Analyses (PRISMA) flow diagram [12].

2.2. Inclusion and Exclusion Criteria. The inclusion criteria were as follows: (1) the study should report the correlation between the aqueous humor levels of mediators or cytokines (VEGF, TNF- $\alpha$, IL-6, IL-8, IL-12, IP-10, and MCP-1) and DR/DME; (2) it should be written in English or Chinese with the full text available; (3) patients with any age, gender, region or race were considered.

Exclusion criteria were predefined as follows: (1) literature reviews, case reports, and cell lines or animal studies; (2) duplication: same studies came from different databases; (3) no sufficient data to perform meta-analysis; (4) no DM group or no health control group.

2.3. Data Extraction. Two reviewers (Lei Liu and Jingyang $\mathrm{Wu}$ ) independently reviewed each included study. The disagreements on eligibility during the reviewing were discussed and resolved by the third reviewer (Song Yue). The following data were extracted by three reviewers (Yifan Zhong, Kaibo Yang, and Song Yue): the first author, location, year of publication, number of subjects in DR, DME, and control groups, definition of DR, cytokine measurements, and name of 


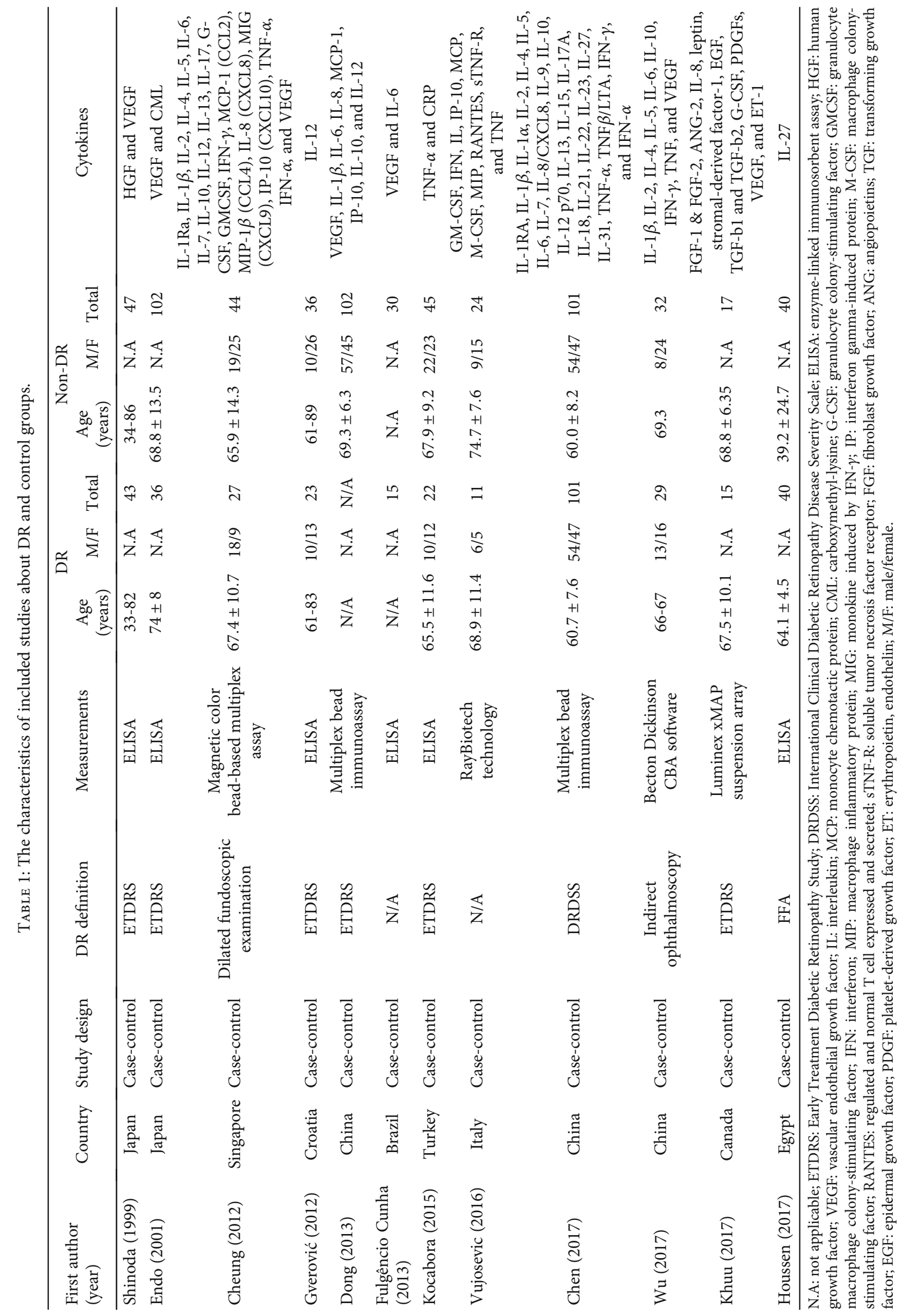


TABLE 2: Assessment of the quality of the included studies using the Newcastle-Ottawa scale (NOS).

\begin{tabular}{|c|c|c|c|c|c|c|c|c|c|}
\hline $\begin{array}{l}\text { First } \\
\text { author }\end{array}$ & $\begin{array}{c}\text { Quality } \\
\text { evaluation }\end{array}$ & $\begin{array}{c}\text { Case } \\
\text { definition }\end{array}$ & Representativeness & $\begin{array}{c}\text { Selection } \\
\text { of controls }\end{array}$ & $\begin{array}{l}\text { Definition } \\
\text { of controls }\end{array}$ & Comparability & $\begin{array}{c}\text { Ascertainment } \\
\text { of exposure }\end{array}$ & $\begin{array}{l}\text { Same } \\
\text { method }\end{array}$ & $\begin{array}{c}\text { Nonresponse } \\
\text { rate }\end{array}$ \\
\hline Shinoda & 6 & 1 & 1 & 1 & 1 & 0 & 1 & 1 & 0 \\
\hline Endo & 6 & 1 & 1 & 1 & 1 & 0 & 1 & 1 & 0 \\
\hline Funk & 7 & 1 & 1 & 1 & 1 & 1 & 1 & 1 & 0 \\
\hline $\mathrm{Oh}$ & 6 & 1 & 1 & 1 & 1 & 0 & 1 & 1 & 0 \\
\hline Lee & 7 & 1 & 1 & 1 & 1 & 1 & 1 & 1 & 0 \\
\hline Cheung & 6 & 1 & 1 & 1 & 1 & 0 & 1 & 1 & 0 \\
\hline Jonas & 7 & 1 & 1 & 1 & 1 & 1 & 1 & 1 & 0 \\
\hline $\begin{array}{l}\text { Gverović } \\
\text { Antunica }\end{array}$ & 6 & 1 & 1 & 1 & 1 & 0 & 1 & 1 & 0 \\
\hline Umazume & 6 & 1 & 1 & 1 & 1 & 0 & 1 & 1 & 0 \\
\hline Dong & 7 & 1 & 1 & 1 & 1 & 1 & 1 & 1 & 0 \\
\hline $\begin{array}{l}\text { Fulgêncio } \\
\text { Cunha }\end{array}$ & 6 & 1 & 1 & 1 & 1 & 0 & 1 & 1 & 0 \\
\hline Kocabora & 7 & 1 & 1 & 1 & 1 & 1 & 1 & 1 & 0 \\
\hline Vujosevic & 7 & 1 & 1 & 1 & 1 & 1 & 1 & 1 & 0 \\
\hline Chen & 7 & 1 & 1 & 1 & 1 & 1 & 1 & 1 & 0 \\
\hline $\mathrm{Wu}$ & 7 & 1 & 1 & 1 & 1 & 1 & 1 & 1 & 0 \\
\hline Noma & 6 & 1 & 1 & 1 & 1 & 0 & 1 & 1 & 0 \\
\hline Khuu & 7 & 1 & 1 & 1 & 1 & 1 & 1 & 1 & 0 \\
\hline Houssen & 7 & 1 & 1 & 1 & 1 & 1 & 1 & 1 & 0 \\
\hline
\end{tabular}

cytokines in detail. We defined that diabetic patients without retinopathy and/or matched healthy persons constituted the controls and patients with DR or DME were the cases.

2.4. Assessment of Methodology Quality. The quality of the included studies was assessed using the Newcastle-Ottawa scale (NOS) [13]. A quality score of more than or equal to seven on the nine-point NOS was considered to be high quality for included studies.

2.5. Statistical Analysis. The weighted standardized mean difference (SMD) was measured with 95\% confidence interval (CI) using random-effects method. The subgroup analyses were conducted based on location, DR definition, and cytokine measurements. $I^{2}$ tests were used to evaluate the statistical heterogeneity among the included studies, and the heterogeneity was considered statistical when $P<0.05$ and $I^{2} \geq 50 \%$. Additionally, sensitive analysis was also performed to evaluate the influences of individual studies on the final effect. The funnel plots were performed to evaluate the potential publication bias. This meta-analysis was performed using the Stata 11.0 statistical software (Stata Corp., College Station, TX). A two-tailed $P$ less than 0.05 was considered as significant difference.

\section{Results}

3.1. Literature Search. The study selection flowchart was shown in Figure 1. A total of 3,045 articles were identified from the databases (PubMed, Embase, Web of Science, CNKI, and Wanfang databases). 1,650 articles were excluded based on a review of the titles and abstracts, and 1,261 articles were excluded as duplicates, review, letter, and editorials. In addition, 108 articles were excluded as not human studies or case-control studies, and 53 articles were excluded as missing sufficient data. Finally, a total of 18 articles $[7,10,11,14-28]$ were included in this meta-analysis.

3.2. Characteristics of the Studies. 18 case-control studies involving 362 cases with DR including 100 cases with DME and 620 controls with type 2 diabetes but without DR were included in the current meta-analysis. The characteristics of the included studies were presented in Table 1 and Table S1. All cited references measured cytokines in different ways (e.g., Luminex xMAP suspension array, ELISA, and multiplex bead immunoassay). As shown in Table 2, according to the NOS checklist, 10 studies with scores $\geq 7$ stars were considered high quality, and the remaining eight studies were medium quality for 6 stars.

\subsection{Meta-Analysis and Subgroup and Sensitivity Analysis}

3.3.1. Analysis of VEGF. Figure 2(a) shows the pooled SMD derived from all 8 studies with the levels of VEGF in the aqueous humor of subjects with and without DR. The results present that there was a significant difference (SMD 1.94, 95\% CI $(1.05,2.83))$ but with heterogeneity in the aqueous humor level of VEGF between the DR and control groups. Further, the publication bias was not significant (Figure 2(b)).

Bending with significant heterogeneity, it was necessary to perform subgroup and sensitivity analyses. Location, DR 


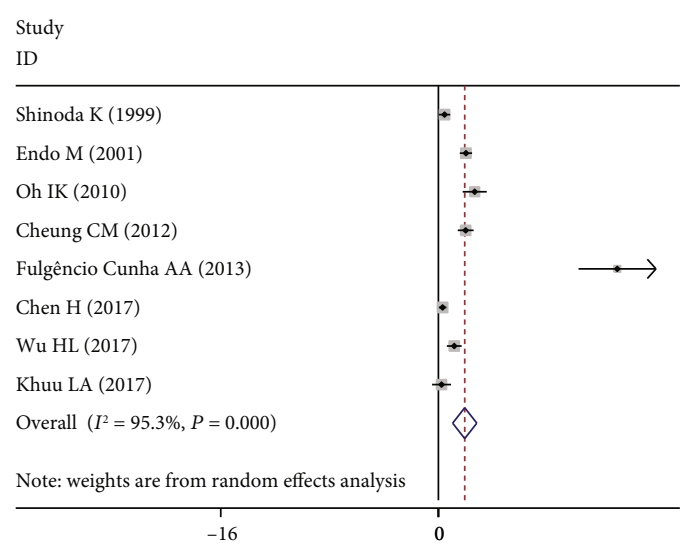

(a)

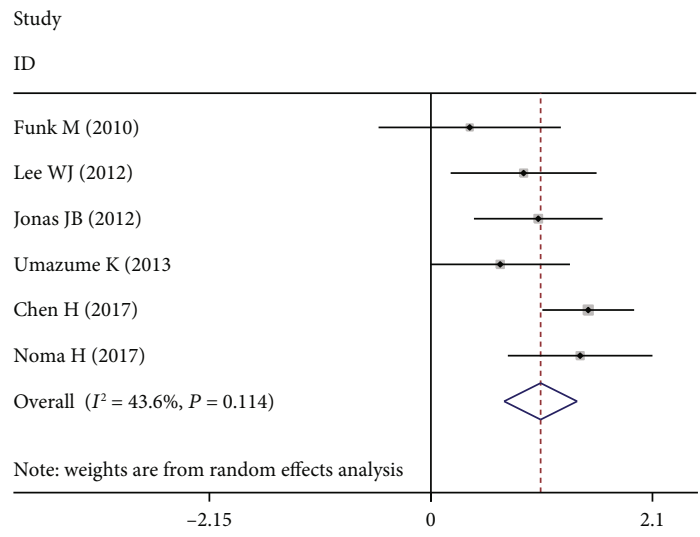

(c)

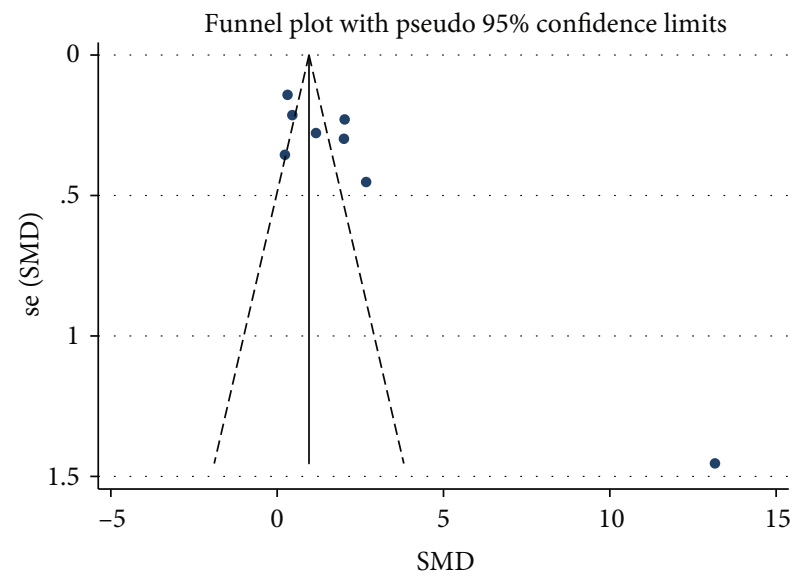

(b)

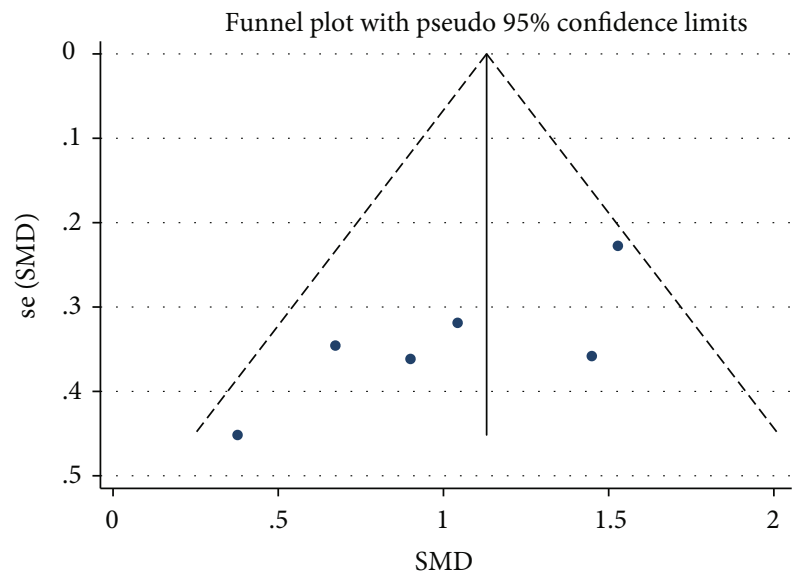

(d)

Figure 2

definition, and cytokine measurements may be the main causes of heterogeneity, but heterogeneity was still significant $\left(I^{2}=93.4 \%, 93.1 \%\right.$, and $97.8 \%$, respectively) after the subgroup analysis (Table 3 ). A sensitivity analysis was used to evaluate the stability and reliability of the results (Figure 3 ). After removing the studies $[11,14,21]$ that were contributing the most to the heterogeneity, the results did not change substantially (SMD 1.61, 95\% CI $(0.89,2.33), P<0.001$, $\left.I^{2}=85.8 \%\right)$.

There were significant differences in the aqueous humor level of VEGF between the DME and controls with SMD 1.07 (95\% CI (0.71, 1.42), $P<0.001$, Figures 2(c) and 2(d)) and no significant heterogeneity $\left(I^{2}=43.6 \%, P=0.114\right)$. Moreover, the funnel plot showed that publication bias was also not significant.

3.3.2. Analysis of IL-6. There were 3 studies that reported data on the relationship of IL-6 levels and DR among participants with T2DM. The results present that there were significant differences between DR and control groups (SMD 3.53, $95 \%$ CI $(0.37,6.69), P=0.028)$, but with significant heterogeneity (Figure 4(a)). However, the publication bias was not significant (Figure 4(b)).
The results of IL- 6 levels between the DME and control groups were shown in Figures 4(c) and 4(d). The pooled SMD was 1.26 , and the $95 \%$ CI was 0.30 to $2.21(P=0.010$, Figure $4(\mathrm{c}))$. The results present that there were significant differences but heterogeneity $\left(I^{2}=86.4 \%, P=0.001\right)$ between these two groups. Subgroup analysis showed that cytokine measurements were the reason for obvious heterogeneity $\left(I^{2}=0.0 \%, P=0.595\right)$. Performing subgroup analysis according to the cytokine measurements, the association was still significant (SMD 0.79, 95\% CI $(0.31,1.27), P=0.001$ ). The funnel plot showed that publication bias was not significant (Figure 4(d)).

3.3.3. Analysis of MCP-1. Compared to the controls, the DR patients resulted in significantly increased MCP-1 levels (SMD 0.49; 95\% CI (0.09-0.89), $P=0.017$ ) in aqueous humor (Figure 5(a)) with no significant heterogeneity between studies $\left(I^{2}=0.0 \%, P=0.764\right)$. The publication bias was not significant (Figure 5(b)).

There were 4 studies that were included in the metaanalysis for the association of MCP-1 levels with the risk of DME. The pooled SMD for DME was 1.49 (95\% CI (0.78-2.20), $P<0.001$, Figure 5(c)) while with substantial heterogeneity among studies $\left(I^{2}=63.9 \%, P=0.040\right)$. Subgroup 
TABLE 3: Subgroup analyses according to the location, diagnosis criteria of DR, and cytokine measurements.

\begin{tabular}{|c|c|c|c|c|c|}
\hline & Number of studies & $\operatorname{SMD}(95 \% \mathrm{CI})$ & $P$ & $I^{2}$ & $P^{*}$ \\
\hline \multicolumn{6}{|l|}{ Location } \\
\hline Asia & 6 & $1.40(0.65,2.14)$ & $<0.001$ & $93.40 \%$ & $<0.001$ \\
\hline South America & 1 & $13.16(10.31,16.01)$ & $<0.001$ & N.A & N.A \\
\hline North America & 1 & $0.23(-0.46,0.93)$ & 0.513 & N.A & N.A \\
\hline \multicolumn{6}{|l|}{ Diagnosis criteria for DR } \\
\hline ETDRS & 5 & $1.32(0.25,2.40)$ & 0.016 & $93.10 \%$ & $<0.001$ \\
\hline Dilated fundoscopic examination & 1 & $2.01(1.42,2.59)$ & $<0.001$ & N.A & N.A \\
\hline $\begin{array}{l}\text { International Clinical Diabetic Retinopathy } \\
\text { Disease Severity Scale }\end{array}$ & 1 & $0.31(0.03,0.59)$ & 0.028 & N.A & N.A \\
\hline Indirect ophthalmoscopy & 1 & $1.17(0.62,1.71)$ & $<0.001$ & N.A & N.A \\
\hline \multicolumn{6}{|l|}{ Cytokine measurements } \\
\hline ELISA & 3 & $4.26(1.76,6.75)$ & 0.001 & $97.80 \%$ & $<0.001$ \\
\hline Luminex xMAP suspension array & 1 & $0.23(-0.46,0.93)$ & 0.513 & N.A & N.A \\
\hline Multiplex bead array assay & 1 & $2.67(1.79,3.56)$ & $<0.001$ & N.A & N.A \\
\hline Magnetic color bead-based multiplex assay & 1 & $2.01(1.42,2.59)$ & $<0.001$ & N.A & N.A \\
\hline Multiplex bead immunoassay & 1 & $0.31(0.03,0.59)$ & 0.028 & N.A & N.A \\
\hline Becton Dickinson CBA software & 1 & $1.17(0.62,1.71)$ & $<0.001$ & N.A & N.A \\
\hline
\end{tabular}

$* P$ value for heterogeneity. SMD: standardized mean difference; ETDRS: Early Treatment Diabetic Retinopathy Study; N.A: not applicable; ELISA: enzymelinked immunosorbent assay.

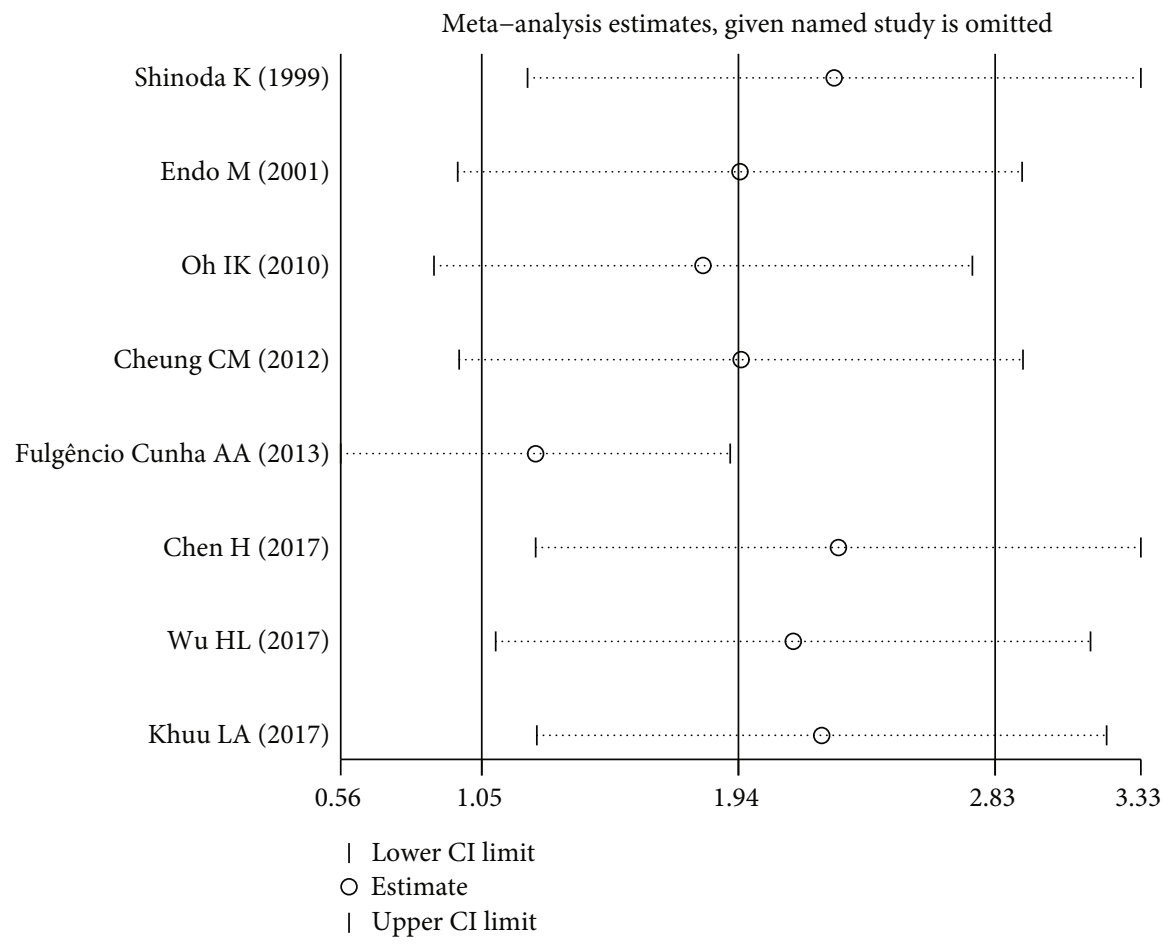

Figure 3

analysis was performed according to the cytokine measurements (SMD 1.13, 95\% CI $(0.69,1.57), P<0.001)$, and the heterogeneity was not significant $\left(I^{2}=0.0 \%, P=0.660\right)$. The funnel plot showed that publication bias was not significant (Figure 5(d)).
3.3.4. Analysis of $I L-8$. The pooled SMD was 0.38 (95\% CI (-0.05-0.81), $P=0.087$ ) for the association between IL-8 levels in the aqueous humor and risk for DR (Table 4). Moreover, the funnel plot showed that publication bias was not significant. 


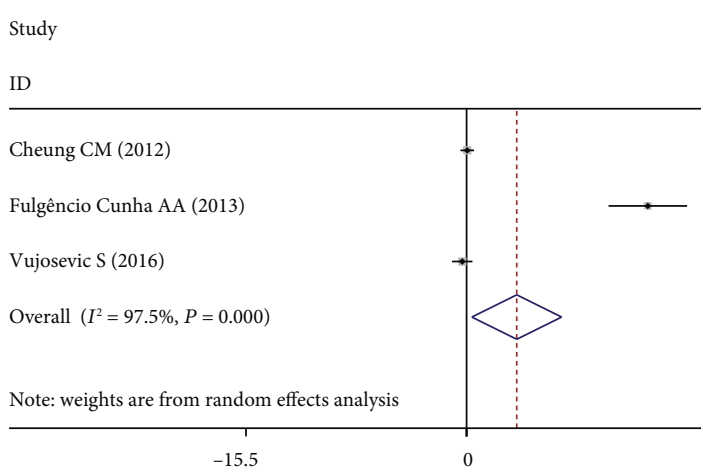

(a)

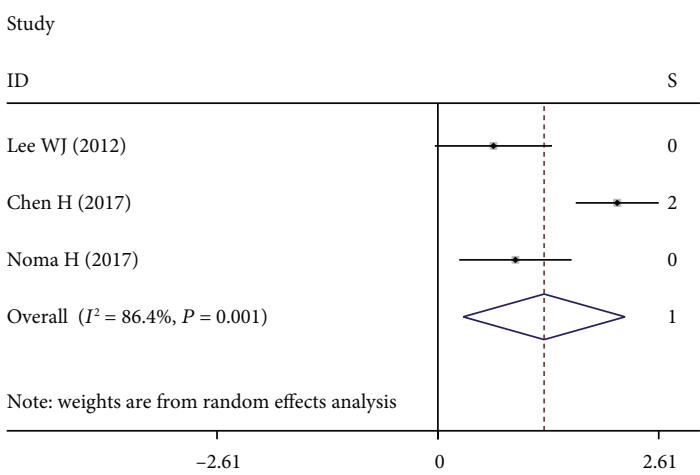

(c)

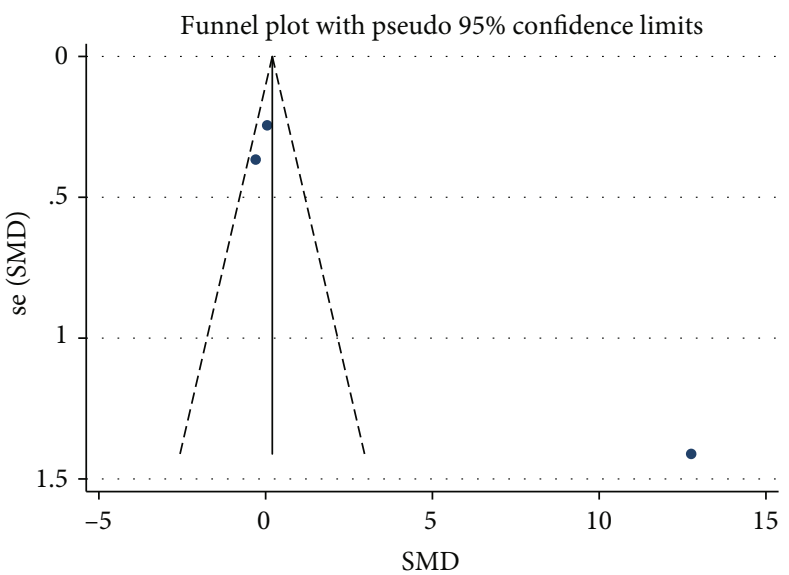

(b)

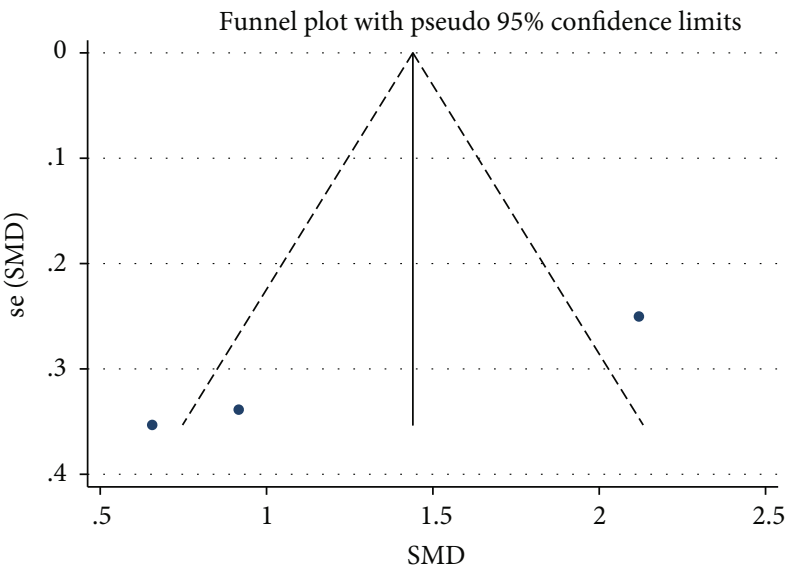

(d)

Figure 4

On the basis of the five studies, the overall SMD of DME was 1.68 (95\% CI $(0.97-2.40), P<0.001)$ in IL-8 levels (Figure 6(a)). However, there was a significant heterogeneity among the five studies $\left(I^{2}=81.0 \%, P<0.001\right)$. Dividing into subgroups according to countries, definition of DR, and cytokine measurements, respectively, heterogeneity was still evident (Table 4). After removing the study by Noma et al. [17], which was contributing to the significant heterogeneity, the heterogeneity was not significant $\left(I^{2}=0.0 \%, P=0.525\right)$. The funnel plot showed that publication bias was not significant (Figure 6(b)).

3.3.5. Analysis of IL-12. Three original studies were included in the current meta-analysis for the association of IL-12 levels with the risk of DR. Finally, the overall SMD of DR was 0.44 (95\% CI (-0.81-1.70), $P=0.488)$ in IL-12 levels (Table 4). The publication bias was not significant.

3.3.6. Analysis of IP-10. Two studies were included in the meta-analysis for the association of IP-10 level with the risk of DR. The pooled SMD for DR was 0.31 (95\% CI $(-0.16,0.77), P=0.193$, Table 4$)$ with substantial heterogeneity between studies $\left(I^{2}=20.5 \%, P=0.262\right)$. The publication bias was not significant.
3.3.7. Analysis of TNF- $\alpha$. There were 4 studies which involved the aqueous humor levels of TNF- $\alpha$ and its risk for DR. Table 4 also shows that the pooled SMD of TNF- $\alpha$ for DR was $0.51(95 \% \mathrm{CI}(-0.04-1.06), P=0.067)$ and without significant publication bias.

\section{Discussion}

To the best of our knowledge, the present meta-analysis evaluates the influence of the aqueous humor concentration of inflammatory cytokines (IL-6 among ten studies, IL-8 among ten studies, IL-10 among six studies, IL-12 among seven studies, TNF- $\alpha$ among six studies, MCP- 1 among nine studies, and VEGF among thirteen studies) on the risk of DR or DME. In the current meta-analysis, we found that elevated levels of mediators or cytokines (VEGF, IL-6, and MCP-1) in the aqueous humor were strongly associated with $\mathrm{DR}$, while the levels of VEGF, IL-6, IL-8, and MCP-1 were strongly associated with DME. Moreover, our meta-analysis presents strong points: the literature review was updated to comprise the latest evidence; a set of inclusion and exclusion criteria were applied for study selection; and outcomes were robust in the sensitivity or subgroup analysis. 


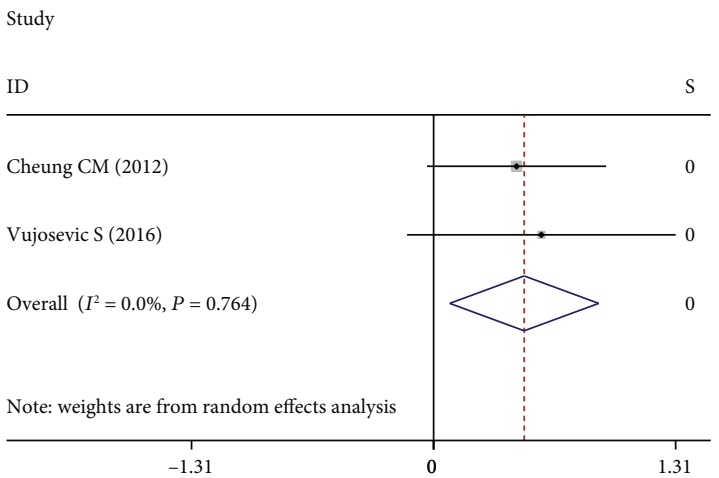

(a)

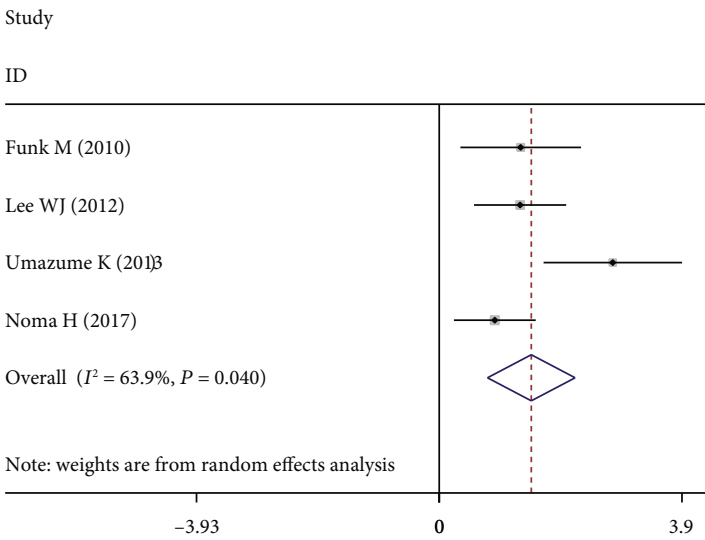

(c)

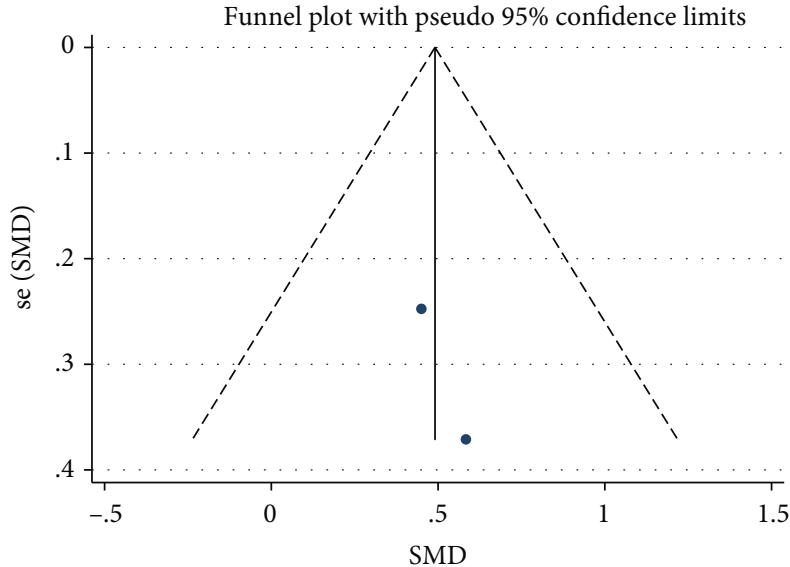

(b)

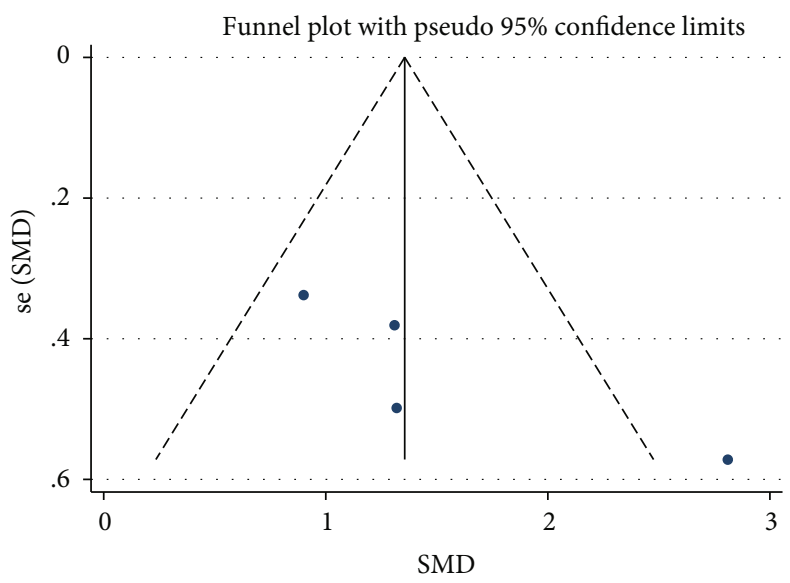

(d)

Figure 5

In the current meta-analysis, there was a significant association between the aqueous humor VEGF levels and the risk for both DR and DME, and similar outcomes were also found in previous studies by $\mathrm{Wu}$ et al. [15] and Endo et al. [25]. VEGF is an endothelial cell mitogen, which can induce increases in vascular permeability and angiogenesis, enhance collateral vessel formation, and increase the permeability of the microvasculature [29]. Moreover, previous studies have pointed out that the VEGF level was significantly correlated with the severity of DR [30]. Recently, anti-VEGF drug intraventricular injection had been commonly used for the treatment of DR and DME [31,32]. However, there are some complications associated with intravitreal injection, such as increased intraocular pressure (IOP) [33], endophthalmitis [34], and geographic atrophy [35]. Our results confirmed that VEGF levels in the aqueous humor have a significant correlation with both DR and DME. Therefore, the dosage form of VEGF may be changed to avoid the above complications and achieve the same therapeutic effect.

Another interesting finding of this meta-analysis is that levels of IL-6 in the aqueous humor have a significant relationship with DR and DME. It is well known that continuous proinflammatory responses and neovascularization are related to the occurrence and progression of DR. IL-6 is a proinflammatory cytokine and is a key factor in host defense against environmental stress such as inflammation, infection, and injury [36]. Moreover, IL-6 can also increase vascular permeability and angiogenesis by inducing VEGF expression [37]. Furthermore, Arjamaa et al. have documented that increased levels of IL-6 in the vitreous significantly correlated with the activity of neovascularization [38]. Furthermore, IL-6 could not only enhance inflammation responses of DR or DME but also contribute to neovascularization and promote the reaction with VEGF at the same time.

MCP-1 is also a proinflammatory cytokine, which can induce monocyte and macrophage infiltration into tissues and trigger their transmigration to the sites of inflammation produced by tissue hypoxia, infection, or macrophage injury [39-42]. Several studies reported that vitreous or aqueous levels of MCP-1 were higher in the eyes with DR compared with normal controls [7,9]. They suggested that MCP-1 may play as a mediator in capillary occlusion in DR through the activation and adhesion of leukocytes and macrophages to the endothelium. Moreover, vitreous MCP-1 levels were found significantly correlated with the degree of proliferative membrane in the eyes with proliferative diabetic retinopathy (PDR), suggesting that MCP-1 may play an important role in 
TABLE 4: The associations between IL- 8 with DR and DME and IL-12, IP10, and TNF- $\alpha$ with DR.

\begin{tabular}{|c|c|c|c|c|c|}
\hline & Number of studies & $\operatorname{SMD}(95 \% \mathrm{CI})$ & $P$ & $I^{2}$ & $P^{*}$ \\
\hline \multicolumn{6}{|l|}{ IL-8 \& DR } \\
\hline Total & 3 & $0.38(-0.05,0.81)$ & 0.078 & $60.80 \%$ & 0.087 \\
\hline IL-8 \& DME & 5 & $1.68(0.97,2.40)$ & $<0.001$ & $81.00 \%$ & $<0.001$ \\
\hline \multicolumn{6}{|l|}{ Subgroup } \\
\hline \multicolumn{6}{|l|}{ Countries } \\
\hline Asian & 4 & $1.86(0.95,2.77)$ & $<0.001$ & $83.90 \%$ & $<0.001$ \\
\hline European & 1 & $1.08(0.45,1.71)$ & 0.001 & N.A & N.A \\
\hline \multicolumn{6}{|l|}{$D R$ diagnosis criteria } \\
\hline ETDRS & 3 & $2.05(0.61,3.49)$ & 0.005 & $88.90 \%$ & $<0.001$ \\
\hline Diabetic Retinopathy Disease Severity Scale & 1 & $1.49(1.04,1.93)$ & $<0.001$ & N.A & N.A \\
\hline \multicolumn{6}{|l|}{ Cytokine measurements } \\
\hline Luminex xMAP suspension array & 3 & $1.84(0.42,3.25)$ & 0.011 & $90.50 \%$ & $<0.001$ \\
\hline BD Cytometric Bead Array & 1 & $1.61(0.68,2.54)$ & 0.001 & N.A & N.A \\
\hline Multiplex bead immunoassay & 1 & $1.49(1.04,1.93)$ & $<0.001$ & N.A & N.A \\
\hline \multicolumn{6}{|l|}{ Remove each study for sensitivity analysis } \\
\hline Lee et al., 2012 & 4 & $1.87(1.0,2.74)$ & $<0.001$ & $83.90 \%$ & $<0.001$ \\
\hline Jonas et al., 2012 & 4 & $1.86(0.95,2.77)$ & $<0.001$ & $83.90 \%$ & $<0.001$ \\
\hline Umazume et al., 2013 & 4 & $1.71(0.85,2.58)$ & $<0.001$ & $85.70 \%$ & $<0.001$ \\
\hline Chen et al., 2017 & 4 & $1.77(0.72,2.82)$ & 0.001 & $85.70 \%$ & $<0.001$ \\
\hline Noma et al., 2017 & 4 & $1.32(1.01,1.62)$ & $<0.001$ & $0 \%$ & 0.525 \\
\hline \multicolumn{6}{|l|}{ IL-12 \& DR } \\
\hline Total & 3 & $0.44(-081,1.70)$ & 0.488 & $94.20 \%$ & $<0.001$ \\
\hline \multicolumn{6}{|l|}{ IP-10 \& DR } \\
\hline Total & 2 & $0.31(-0.16,0.77)$ & 0.017 & $20.50 \%$ & 0.262 \\
\hline \multicolumn{6}{|l|}{ TNF- $\alpha \& \mathrm{DR}$} \\
\hline Total & 4 & $0.51(-0.04,1.06)$ & 0.067 & $80.20 \%$ & 0.002 \\
\hline
\end{tabular}

${ }^{*} P$ value for heterogeneity.

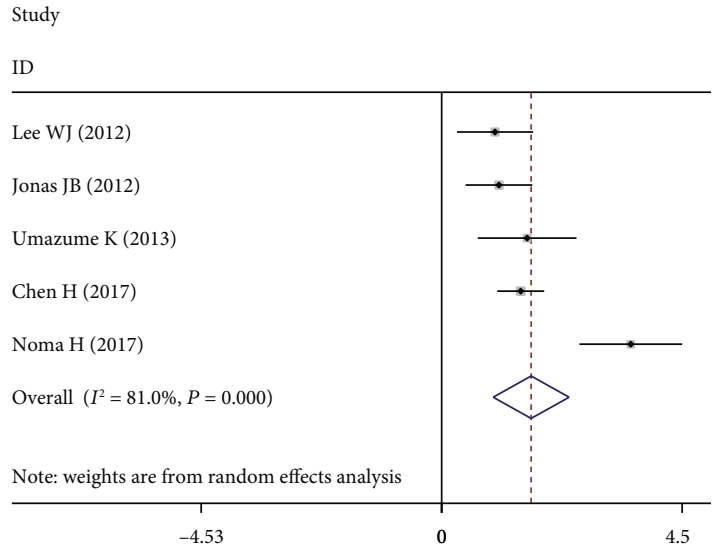

(a)

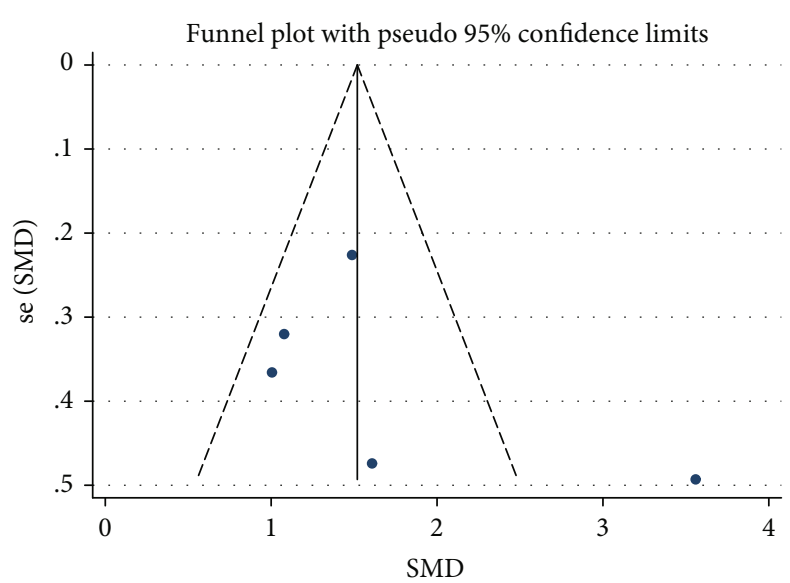

(b)

Figure 6

the development of PDR [43]. In the current meta-analysis, due to the little information on the subtype of DR, we did not perform a meta-analysis on a different type of DR. Fur- ther studies are still needed to evaluate the association regarding cytokines in the aqueous humor and its risk for the severity of DR. 
Moreover, previous studies have reported that the aqueous levels of MCP-1 were significantly higher in DME patients than in controls $[8,14]$. Furthermore, the aqueous humor level of MCP-1 was significantly correlated with central macular thickness (CMT), suggesting that MCP-1 might play a role in the development of DME. MCP-1 is a potent eosinophil chemotactic cytokine and could recruit the monocytes to the sites of vascular injury [44]. Meanwhile, the monocytes and macrophages which accumulate on the wall of the blood vessel increase vascular permeability that potentiate DME [45]. In addition, the activity of MCP-1 seems to be closely related to VEGF because MCP-1 could mediate the gene expression of VEGF-A [46].

Indeed, IL- 8 is the prototype of a CXC chemokine, which has been recognized as a potent chemoattractant and an activator of neutrophils and T lymphocytes [41, 47]. It is induced by hypoxia in vascular endothelial cells and plays an important role in promoting angiogenesis and tumor metastasis [41]. Once, some scholars reported that the levels of IL-8 in the vitreous were higher in the eyes with active PDR compared with quiescent PDR and they suggested that IL-8 might play an important part in the pathogenic process of PDR [48]. Elner et al. [49] also pointed out that the levels of IL-8 in the vitreous were significantly increased in active PDR. In our meta-analysis, we did not find any relationship between IL- 8 and DR, maybe because we did not classify DR by severity. Moreover, researchers reported that IL- 8 directly stimulates VEGF expression and the autocrine activation of VEGF receptor- (VEGFR-) 2 in vascular endothelial cells [19]. Kaneda et al. [50] reported that IL-8 appears to be involved in angiogenesis, endothelial cell binding and regeneration, endothelial wound healing, and vascular remodeling, presumably together with VEGF. These may be the reason why the aqueous humor levels of IL- 8 increased significantly in the patients with DME.

Although some studies revealed that there are potential associations between the aqueous levels of IL-12, IP-10, or TNF- $\alpha$ and DR $[15,24]$, other outcomes regarding those associations are controversial [14, 20, 26, 27]. In our metaanalysis, there was no significant correlation between these cytokines and DR. Due to the lack of sufficient data, the association between cytokines (IL-12, IP-10, and TNF- $\alpha$ ) and $\mathrm{DME}$ was not evaluated. Hence, further researches are needed for the evidence-based consequence.

The strengths of the current study include the comprehensive exploration of the evidence on the association between the cytokines in the aqueous humor and the risk of both DR and DME. However, there were some limitations in our study. First, there was a lack of studies available on the severity of DR for comparisons. Hence, our metaanalysis could only incorporate studies regarding any DR and controls. Second, we cannot obtain powerful outcomes adjusting potential confounders between the levels of cytokines and DR although we have done the subgroup analysis based on whether included studies had differences between case and control group or not. Last but not the least, there were insufficient studies for some cytokines such as IL-12, IP-10, and TNF- $\alpha$ to provide enough evidences to demonstrate the association between the biomarkers and risk for both DR and DME. Hence, large-sample, longitudinal, population-based studies are needed to validate our findings in the future.

\section{Conclusion}

In conclusion, we reviewed the literatures and conducted a current comprehensive meta-analysis to evaluate the association between the aqueous humor levels of VEGF, TNF- $\alpha$, and inflammatory cytokines and risk for both DR and DME. Our findings indicated that lower mediator or cytokine levels in the aqueous humor may work best to attenuate DR and DME risk. This meta-analysis was the first comprehensive quantitative assessment of the aqueous humor levels of VEGF, IL-6, and MCP-1 on DR, which suggested that they may be used as biomarkers of the occurrence or development of $\mathrm{DR}$, and reminded a new treatment besides intravitreal injection.

\section{Data Availability}

The data used to support the findings of this study are included within the article.

\section{Disclosure}

The funders had no involvement in the study design, data collection and analysis, decision to publish, or preparation of the manuscript.

\section{Conflicts of Interest}

The authors declare that they have no conflict of interests.

\section{Authors' Contributions}

Jingyang $\mathrm{Wu}$ and Yifan Zhong are co-first authors to this manuscript.

\section{Acknowledgments}

This article is supported by the National Natural Science Foundation of China (No. 81300783), the Department of Education of Liaoning Province (No. LQNK201703), and the Liaoning Revitalization Talents Program (No. XLYC1807082).

\section{Supplementary Materials}

The basic clinical information and characteristics of each included study about diabetic macular edema (DME) on the system. (Supplementary Materials)

\section{References}

[1] N. Cheung, P. Mitchell, and T. Y. Wong, "Diabetic retinopathy," The Lancet, vol. 376, no. 9735, pp. 124-136, 2010.

[2] H. Funatsu, H. Yamashita, H. Noma et al., "Aqueous humor levels of cytokines are related to vitreous levels and progression of diabetic retinopathy in diabetic patients," Graefe's Archive 
for Clinical and Experimental Ophthalmology, vol. 243, no. 1, article 950, pp. 3-8, 2005.

[3] A. M. Abu el Asrar, D. Maimone, P. H. Morse, S. Gregory, and A. T. Reder, "Cytokines in the vitreous of patients with proliferative diabetic retinopathy," American Journal of Ophthalmology, vol. 114, no. 6, pp. 731-736, 1992.

[4] D. A. Vignali, "Multiplexed particle-based flow cytometric assays," Journal of Immunological Methods, vol. 243, no. 1-2, pp. 243-255, 2000.

[5] R. K. Sharma, A. T. Rogojina, and K. V. Chalam, "Multiplex immunoassay analysis of biomarkers in clinically accessible quantities of human aqueous humor," Molecular Vision, vol. 15, pp. 60-69, 2009.

[6] R. Klein, B. Klein, S. Moss, and K. Cruickshanks, "The Wisconsin epidemiologic study of diabetic retinopathy $\mathrm{XV}$ : the long-term incidence of macular edema," Ophthalmology, vol. 102, no. 1, pp. 7-16, 1995.

[7] I. K. Oh, S.-W. Kim, J. Oh, T. S. Lee, and K. Huh, "Inflammatory and angiogenic factors in the aqueous humor and the relationship to diabetic retinopathy," Current Eye Research, vol. 35, no. 12, pp. 1116-1127, 2010.

[8] H. J. Sohn, D. H. Han, I. T. Kim et al., "Changes in aqueous concentrations of various cytokines after intravitreal triamcinolone versus bevacizumab for diabetic macular edema," American Journal of Ophthalmology, vol. 152, no. 4, pp. 686-694, 2011.

[9] Y. Suzuki, M. Nakazawa, K. Suzuki, H. Yamazaki, and Y. Miyagawa, "Expression profiles of cytokines and chemokines in vitreous fluid in diabetic retinopathy and central retinal vein occlusion," Japanese Journal of Ophthalmology, vol. 55, no. 3, pp. 256-263, 2011.

[10] S. Vujosevic, A. Micera, S. Bini, M. Berton, G. Esposito, and E. Midena, "Proteome analysis of retinal glia cells-related inflammatory cytokines in the aqueous humour of diabetic patients," Acta Ophthalmologica, vol. 94, no. 1, pp. 56-64, 2016.

[11] A. A. Fulgêncio Cunha, A. A. Bosco, C. A. Veloso, C. M. Volpe, M. M. Chaves, and J. A. Nogueira-Machado, "Suppressive effect of aqueous humor from person with type 2 diabetes with or without retinopathy on reactive oxygen species generation," Diabetes Research and Clinical Practice, vol. 100, no. 1, pp. 69-73, 2013.

[12] D. Moher, A. Liberati, J. Tetzlaff, D. G. Altman, and The PRISMA Group, "Preferred Reporting Items for Systematic Reviews and Meta-Analyses: The PRISMA Statement," PLoS Medicine, vol. 6, no. 7, article e1000097, 2009.

[13] A. Stang, "Critical evaluation of the Newcastle-Ottawa scale for the assessment of the quality of nonrandomized studies in meta-analyses," European Journal of Epidemiology, vol. 25, no. 9, pp. 603-605, 2010.

[14] H. Chen, X. Zhang, N. Liao, and F. Wen, “Assessment of biomarkers using multiplex assays in aqueous humor of patients with diabetic retinopathy," BMC Ophthalmology, vol. 17, no. 1, p. 176, 2017.

[15] H. Wu, D.-K. Hwang, X. Song, and Y. Tao, "Association between aqueous cytokines and diabetic retinopathy stage," Journal of Ophthalmology, vol. 2017, Article ID 9402198, 8 pages, 2017.

[16] M. Funk, G. Schmidinger, N. Maar et al., "Angiogenic and inflammatory markers in the intraocular fluid of eyes with diabetic MACULAR EDEMA and influence of therapy with bevacizumab," Retina, vol. 30, no. 9, pp. 1412-1419, 2010.
[17] H. Noma, T. Mimura, K. Yasuda, R. Motohashi, O. Kotake, and M. Shimura, "Aqueous humor levels of soluble vascular endothelial growth factor receptor and inflammatory factors in diabetic macular edema," Ophthalmologica, vol. 238, no. 1-2, pp. 81-88, 2017.

[18] L. A. Khuu, F. Tayyari, J. M. Sivak et al., "Aqueous humour concentrations of TGF- $\beta$, PLGF and FGF-1 and total retinal blood flow in patients with early non-proliferative diabetic retinopathy," Acta Ophthalmologica, vol. 95, no. 3, pp. e206-e211, 2017.

[19] W. J. Lee, M. H. Kang, M. Seong, and H. Y. Cho, "Comparison of aqueous concentrations of angiogenic and inflammatory cytokines in diabetic macular oedema and macular oedema due to branch retinal vein occlusion," The British Journal of Ophthalmology, vol. 96, no. 11, pp. 1426-1430, 2012.

[20] C. M. Cheung, M. Vania, M. Ang, S. P. Chee, and J. Li, "Comparison of aqueous humor cytokine and chemokine levels in diabetic patients with and without retinopathy," Molecular Vision, vol. 18, pp. 830-837, 2012.

[21] K. Shinoda, S. Ishida, S. Kawashima et al., "Clinical factors related to the aqueous levels of vascular endothelial growth factor and hepatocyte growth factor in proliferative diabetic retinopathy," Current Eye Research, vol. 21, no. 2, pp. 655$661,2000$.

[22] J. B. Jonas, R. A. Jonas, M. Neumaier, and P. Findeisen, "Cytokine concentration in aqueous humor of eyes with diabetic macular edema," Retina, vol. 32, no. 10, pp. 2150-2157, 2012.

[23] K. Umazume, Y. Usui, Y. Wakabayashi, Y. Okunuki, T. Kezuka, and H. Goto, "Effects of soluble CD14 and cytokine levels on diabetic macular edema and visual acuity," Retina, vol. 33, no. 5, pp. 1020-1025, 2013.

[24] A. G. Antunica, K. Karaman, L. Znaor, A. Sapunar, V. Buško, and V. Puzović, "IL-12 concentrations in the aqueous humor and serum of diabetic retinopathy patients," Graefe's Archive for Clinical and Experimental Ophthalmology, vol. 250, no. 6, pp. 815-821, 2012.

[25] M. Endo, K. Yanagisawa, K. Tsuchida et al., "Increased levels of vascular endothelial growth factor and advanced glycation end products in aqueous humor of patients with diabetic retinopathy," Hormone and Metabolic Research, vol. 33, no. 5, pp. 317-322, 2001.

[26] M. S. Kocabora, M. E. Telli, K. Fazil et al., "Serum and aqueous concentrations of inflammatory markers in diabetic macular edema," Ocular Immunology and Inflammation, vol. 24, no. 5, pp. 549-554, 2016.

[27] M. E. Houssen, M. A. B. El-Hussiny, A. El-Kannishy, D. Sabry, R. El Mahdy, and M. E. Shaker, "Serum and aqueous humor concentrations of interleukin-27 in diabetic retinopathy patients," International Ophthalmology, vol. 38, no. 5, pp. 1817-1823, 2018.

[28] N. Dong, B. Xu, B. Wang, and L. Chu, "Study of 27 aqueous humor cytokines in patients with type 2 diabetes with or without retinopathy," Molecular Vision, vol. 19, pp. 1734-1746, 2013.

[29] L. P. Aiello, R. L. Avery, P. G. Arrigg et al., "Vascular endothelial growth factor in ocular fluid of patients with diabetic retinopathy and other retinal disorders," The New England Journal of Medicine, vol. 331, no. 22, pp. 1480-1487, 1994.

[30] H. Funatsu, H. Yamashita, E. Shimizu, R. Kojima, and S. Hori, "Relationship between vascular endothelial growth factor and 
interleukin-6 in diabetic retinopathy," Retina, vol. 21 , no. 5 , pp. 469-477, 2001.

[31] Writing Committee for the Diabetic Retinopathy Clinical Research Network, J. G. Gross, A. R. Glassman et al., "Panretinal photocoagulation vs intravitreous ranibizumab for proliferative diabetic Retinopathy," JAMA, vol. 314, no. 20, pp. 2137-2146, 2015.

[32] Diabetic Retinopathy Clinical Research Network, J. A. Wells, A. R. Glassman et al., "Aflibercept, bevacizumab, or ranibizumab for diabetic macular edema," The New England Journal of Medicine, vol. 372, no. 13, pp. 1193-1203, 2015.

[33] T. J. Good, A. E. Kimura, N. Mandava, and M. Y. Kahook, "Sustained elevation of intraocular pressure after intravitreal injections of anti-VEGF agents," The British Journal of Ophthalmology, vol. 95, no. 8, pp. 1111-1114, 2011.

[34] T. A. Meredith, C. A. McCannel, C. Barr et al., "Postinjection endophthalmitis in the comparison of age-related macular degeneration treatments trials (CATT)," Ophthalmology, vol. 122, no. 4, pp. 817-821, 2015.

[35] J. E. Grunwald, E. Daniel, J. Huang et al., "Risk of geographic atrophy in the comparison of age-related macular degeneration treatments trials," Ophthalmology, vol. 121, no. 1, pp. 150-161, 2014.

[36] M. Mesquida, A. Leszczynska, V. Llorenç, and A. Adán, "Interleukin-6 blockade in ocular inflammatory diseases," Clinical and Experimental Immunology, vol. 176, no. 3, pp. 301-309, 2014.

[37] C. Holzinger, E. Weissinger, A. Zuckermann et al., "Effects of interleukin-1, -2, -4, -6, interferon-gamma and granulocyte/macrophage colony stimulating factor on human vascular endothelial cells," Immunology Letters, vol. 35, no. 2, pp. 109-117, 1993.

[38] O. Arjamaa, M. Pöllönen, K. Kinnunen, T. Ryhänen, and K. Kaarniranta, "Increased IL-6 levels are not related to NF- $\kappa$ B or HIF- $1 \alpha$ transcription factors activity in the vitreous of proliferative diabetic retinopathy," Journal of Diabetes and its Complications, vol. 25, no. 6, pp. 393-397, 2011.

[39] B. J. Rollins, “Chemokines," Blood, vol. 90, no. 3, pp. 909-928, 1997.

[40] K. Matsushima, C. G. Larsen, G. C. DuBois, and J. J. Oppenheim, "Purification and characterization of a novel monocyte chemotactic and activating factor produced by a human myelomonocytic cell line," The Journal of Experimental Medicine, vol. 169, no. 4, pp. 1485-1490, 1989.

[41] J. J. Oppenheim, C. O. Zachariae, N. Mukaida, and K. Matsushima, "Properties of the novel proinflammatory supergene 'intercrine' cytokine family," Annual Review of Immunology, vol. 9, pp. 617-648, 1991.

[42] B. J. Rollins, A. Walz, and M. Baggiolini, "Recombinant human MCP-1/JE induces chemotaxis, calcium flux, and the respiratory burst in human monocytes," Blood, vol. 78, no. 4, pp. 1112-1116, 1991.

[43] Y. Mitamura, S. Takeuchi, A. Matsuda, Y. Tagawa, Y. Mizue, and J. Nishihira, "Monocyte chemotactic protein-1 in the vitreous of patients with proliferative diabetic retinopathy," Ophthalmologica, vol. 215, no. 6, pp. 415-418, 2001.

[44] A. Schober and A. Zernecke, "Chemokines in vascular remodeling," Thrombosis and Haemostasis, vol. 97, no. 5, pp. 730-737, 2007.
[45] G. H. Tesch, "Role of macrophages in complications of type 2 diabetes," Clinical and Experimental Pharmacology \& Physiology, vol. 34, no. 10, pp. 1016-1019, 2007.

[46] K. Hong, J. Ryu, and K. Han, "Monocyte chemoattractant protein-1-induced angiogenesis is mediated by vascular endothelial growth factor-A," Blood, vol. 105, no. 4, pp. 1405-1407, 2005.

[47] D. D. Taub, M. Anver, J. J. Oppenheim, D. L. Longo, and W. J. Murphy, "T lymphocyte recruitment by interleukin-8 (IL-8). IL-8-induced degranulation of neutrophils releases potent chemoattractants for human $\mathrm{T}$ lymphocytes both in vitro and in vivo," The Journal of Clinical Investigation, vol. 97, no. 8, pp. 1931-1941, 1996.

[48] C. Hernandez, R. Segura, A. Fonollosa, E. Carrasco, G. Francisco, and R. Simó, "Interleukin-8, monocyte chemoattractant protein-1 and IL-10 in the vitreous fluid of patients with proliferative diabetic retinopathy," Diabetic Medicine, vol. 22, no. 6, pp. 719-722, 2005.

[49] S. G. Elner, V. M. Elner, G. J. Jaffe, A. Stuart, S. L. Kunkel, and R. M. Strieter, "Cytokines in proliferative diabetic retinopathy and proliferative vitreoretinopathy," Current Eye Research, vol. 14, no. 11, pp. 1045-1053, 1995.

[50] S. Kaneda, D. Miyazaki, S. Sasaki et al., "Multivariate analyses of inflammatory cytokines in eyes with branch retinal vein occlusion: relationships to bevacizumab treatment," Investigative Ophthalmology \& Visual Science, vol. 52, no. 6, pp. 29822988, 2011. 


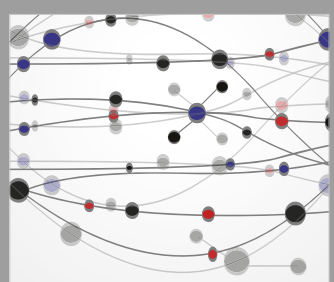

The Scientific World Journal
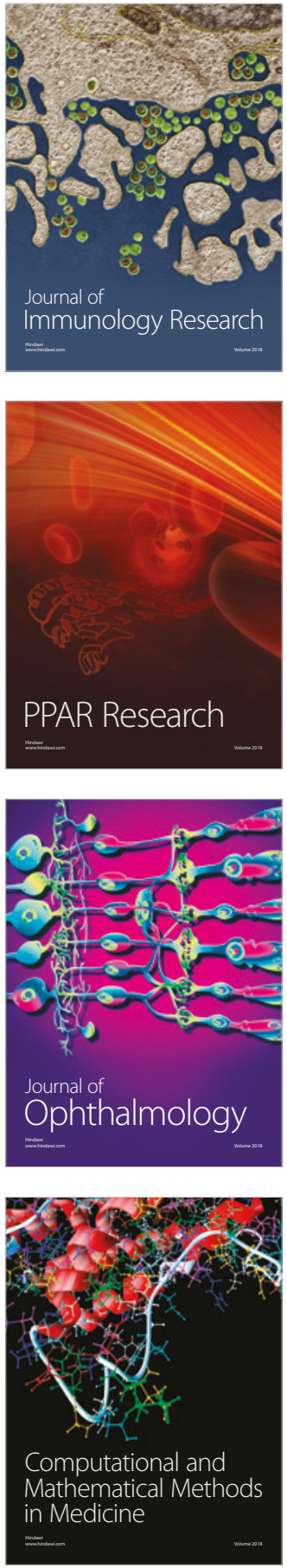

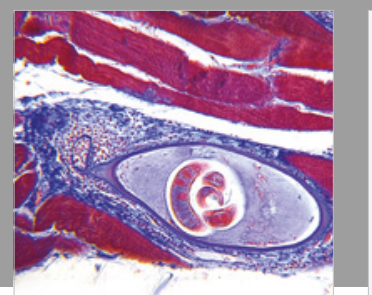

Gastroenterology Research and Practice

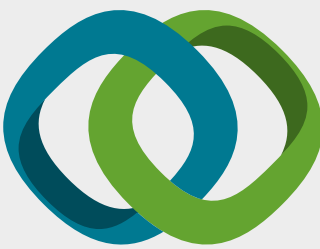

\section{Hindawi}

Submit your manuscripts at

www.hindawi.com
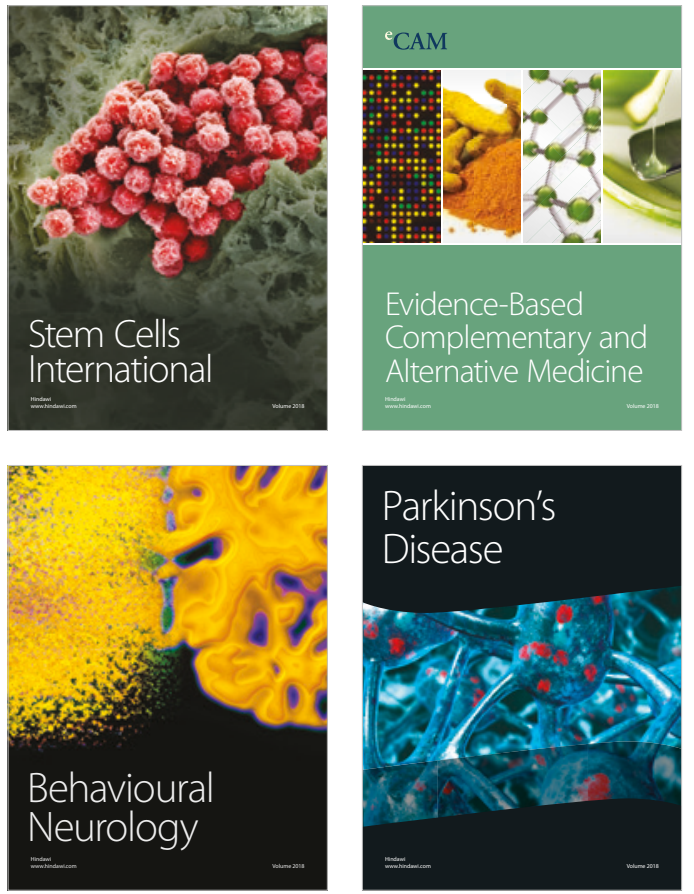

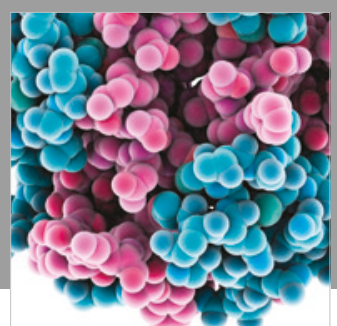

ournal of

Diabetes Research

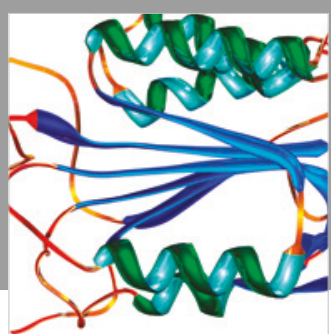

Disease Markers
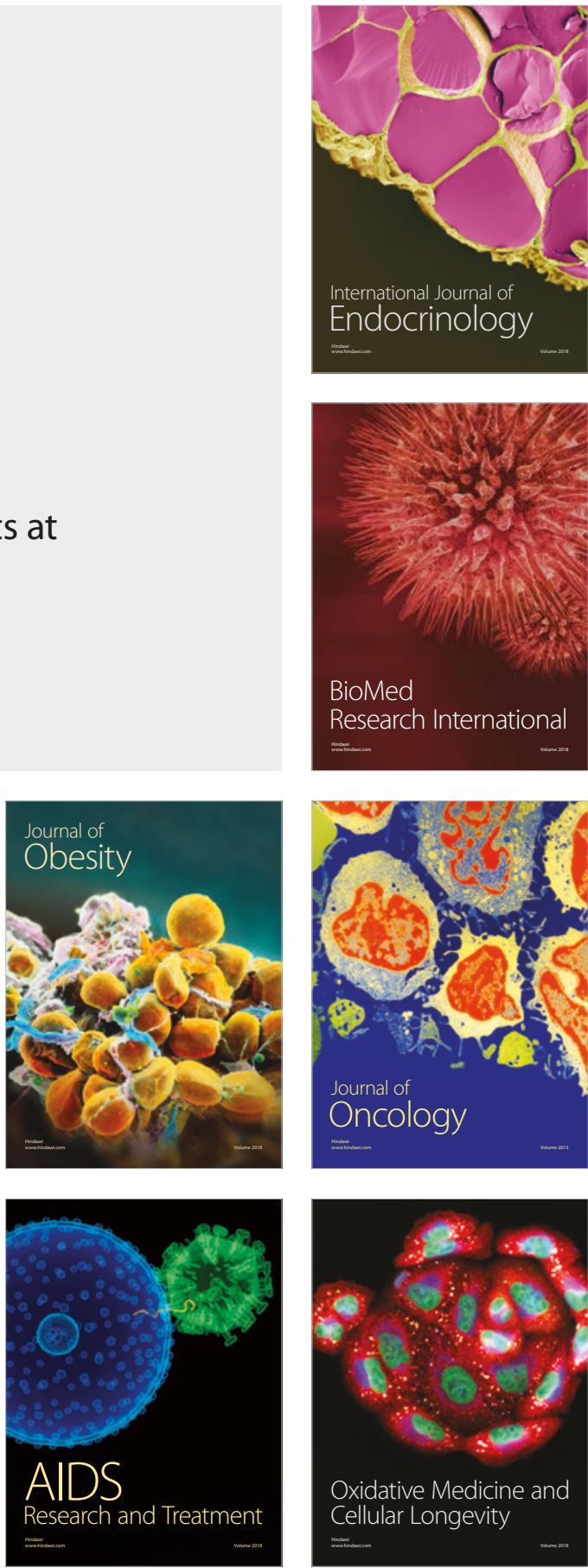